\title{
Kaon Physics: What the Future Holds in Probing the Standard Model and Beyond
}

\section{R. Tschirhart ${ }^{1}$}

Fermi National Accelerator Laboratory

Batavia, Il, USA

E-mail: tsch@fnal.gov

The status and prospects of current and future kaon physics experiments is discussed. Both precision measurements and the search for and measurement of ultra-rare decays are powerful probes of many models of new physics beyond the Standard Model. The physics reach of these experiments is briefly discussed.

\footnotetext{
$1 \quad$ Speaker
} 


\section{Introduction}

Experimental kaon physics remains an active field with the following experiments producing results this year:

\section{E391a at KEK: E949 at BNL: \\ KLOE at Frascati: \\ KTeV at Fermilab: NA62 at CERN:}

\section{Proton beam driven $K_{L}$ decay-in-flight experiment. Proton beam driven $\mathrm{K}^{+}$decay-at-rest experiment. $\mathbf{e}^{+} \mathbf{e}^{-}$storage ring $\phi \rightarrow \mathbf{K}^{+} \mathbf{K}^{-} / \mathbf{K}_{\mathbf{L}} \mathbf{K}_{\mathrm{S}}$ experiment. Proton beam driven $K_{\mathrm{L}}$ decay-in-flight experiment. Proton beam driven $\mathrm{K}$ decay-in-flight experiment.}

These experiments are pursuing measurements to attack the "Flavor Problem" that persists in particle physics today. There are strong theoretical arguments for new physics at the TeV scale, such as Super-Symmetry (SUSY). With generic O(1) couplings this new physics should manifest itself as anomalous quantum corrections to flavor physics phenomena within the Standard Model. The absence of observed anomalous corrections today severely constrains the space of generic couplings...thereby giving rise to the Flavor Problem.

\section{Highlights from the current round of experiments.}

The KLOE experiment this year has substantially improved the measurement of |Vus| by extracting this CKM amplitude from a variety of kaon decay channels in order to control experimental systematic biases ${ }^{1}$. This precision measurement of $|\mathrm{Vus}|$ enables a an incisive test of first-row CKM unitarity which can probe the presence of $4^{\text {th }}$ generations and other new physics. In contrast, assuming CKM unitarity, the effective Fermi coupling $\left(\mathrm{G}_{\mathrm{F}}\right)$ determined from these quark decays can be compared with $\mathrm{G}_{\mathrm{F}}$ determined from muon, tau, and $\mathrm{W} / \mathrm{Z}$ decays. This comparison is thereby sensitive to physics beyond the Standard Model as summarized below in Table 1.

\begin{tabular}{lc}
\hline Source & $\mathbf{G}_{\mathbf{F}}\left(\mathbf{G e V}^{-2}\right) \mathbf{x ~ 1 0}$ \\
\hline From muon decays & $1.166371(6)$ \\
From tau decays & $1.1678 \quad(26)$ \\
From EW precision tests & $1.1655 \quad(12)$ \\
From quarks (KLOE) & $1.16604(40)$ \\
\hline
\end{tabular}

TABLE 1. $G_{\mathrm{F}}$ extracted from various sources ${ }^{1}$. The level of agreement in $\mathrm{G}_{\mathrm{F}}$ extracted from different sources within the Standard Model constrains the contribution from new physics. In models such as $\mathrm{SO}(10)$, corresponding gauge boson $(\mathrm{Z} \chi)$ masses can be limited ${ }^{2}$ to greater than the $10 \mathrm{TeV}$ scale.

The CERN NA62 experiment is pursuing a precision measurement of the ratio $\mathrm{R}=\left(\mathrm{K}^{+} \rightarrow \mathrm{e}^{+} v_{\mathrm{e}}\right) /\left(\mathrm{K}^{+} \rightarrow \mu^{+} v_{\mu}\right)$ which is senstive to new physics. The $\mathrm{K}^{+} \rightarrow \mathrm{e}^{+} v_{\mathrm{e}}$ decay is helicity suppressed to a branching ratio of $1.1 \times 10^{-5}$. SUSY processes, in particular charged Higgs amplitudes can modify this branching ratio by up to the $1-2 \%$ level $^{3}$. The NA62 experiment has collected a sample of more than $100,000 \mathrm{~K}^{+} \rightarrow \mathrm{e}^{+} v_{\mathrm{e}}$ decays 
and has recently report results ${ }^{3}$ on the ratio $\mathrm{R}$ that is consisitent with the Standard Model expectation at the $0.3 \%$ level. This new measurement contributes substantially to furthering constraints on charged Higgs couplings within the context of SUSY.

\section{Expectations for the future program.}

The following experiments and initiatives are preparing or considering major upgrades:

\section{E14 at JPARC: $\quad$ Proton beam driven $K_{\mathrm{L}}$ decay-in-flight experiment. NA62 at CERN: $\quad$ Proton beam driven $\mathrm{K}^{+}$decay-in-flight experiment. $\mathrm{K}$-initiative at Fermilab: Proton beam driven $\mathrm{K}^{+}$decay experiment.}

A high precision measurement of the ultra-rare $K^{+} \rightarrow \pi^{+} v \bar{v}$ decay at CERN and or Fermilab would be one of the most incisive probes of quark flavor physics in the coming decade. The CERN experiment, NA62, is a major upgrade of the existing detector and beam-line systems and proposes a sensitivity of about 100 Standard Model events. An initiative at Fermilab is studying experiment concepts with a sensitivity goal of 1000 Standard Model events.

The dramatic physics reach of a precision measurement of $K^{+} \rightarrow \pi^{+} v \bar{\nu}$ is due to three factors:

1) The Standard Model prediction for the $K^{+} \rightarrow \pi^{+} v \bar{v}$ and $K_{L}^{0} \rightarrow \pi^{0} v \bar{v}$ branching fractions are broadly recognized as theoretically robust to the $2-4 \%$ level. No other loop-dominated quark process can be predicted with this level of certainty.

2) The $K^{+} \rightarrow \pi^{+} v \bar{v}$ branching fraction is highly suppressed in the Standard Model with an expected branching fraction of $7 \times 10^{-11}$. This suppression allows physics beyond the Standard Model to contribute noticeably to the branching fraction with enhancements of up to factors of x3 above the Standard Model level.

3) The certainty with which the Standard Model contribution to $K^{+} \rightarrow \pi^{+} \nu \bar{\nu}$ is known permits a $5 \sigma$ discovery potential for new physics even for enhancements of the branching fraction as small as $25 \%$.

This sensitivity is unique in quark flavor physics and probes essentially all models of new physics that couple to quarks within the reach of the LHC. Further, precision measurement of $K^{+} \rightarrow \pi^{+} v \bar{v}$ is sensitive to many models of new physics far beyond the direct mass reach of the LHC.

The experimental challenge of measuring $K^{+} \rightarrow \pi^{+} v \bar{v}$ at the 1 in 10-billion Standard Model rate has been met successfully. Several events of the $K^{+} \rightarrow \pi^{+} v \bar{v}$ process have been clearly observed by BNL E949 using the stopped $\mathrm{K}^{+}$technique. The NA62 experiment is pursuing a new technique based on in-flight decays. The Fermilab initiative is now studying the optimal concept for a 1000-event experiment. 
The E14 experiment at JPARC in Tokai Japan is pursuing sensitivity of the $K_{L}^{0} \rightarrow \pi^{0} v \bar{v}$ process at the single Standard Model event level, which would be sensitive to several models beyond the Standard Model. This nearly x100 increase in sensitivity is achieved though upgrading the existing E391 detector and an new high intensity beam-line at the JPARC accelerator complex.

\section{Summary}

Measurements in the kaon system have contributed greatly to establishing both the framework and the details of the Standard Model. The broad sweep of consistency of all flavor physics phenomena today within the Standard Model has led us to the Flavor Problem, which is a steadily growing tension between the expectations of $\mathrm{TeV}$-scale new physics and the apparent absence of corresponding quantum corrections that should affect quark flavor measurements at the current state of the art. The next round of experiments in kaon physics are aimed squarely at the $K^{+} \rightarrow \pi^{+} v \bar{v}$ and $K_{L}^{0} \rightarrow \pi^{0} v \bar{v}$ processes, which are particularly promising tools to crack the Flavor Problem. Proton beam facilities exist world-wide today to mount these experiments which have the potential to deliver these incisive measureents within the coming decade.

\section{References}

[1] T. Spadora, Renconstres de Moriond, March 112009.

[2] B. Marciano (private communication)

[3] Evgueni Goudzovski, KAON-2009 conference at KEK, Tskuba Japan, June 2009. 